\title{
Sociality affects mutational spectrum of mtDNA in termites versus cockroaches
}

\author{
Alina A. Mikhailova \\ Center for Mitochondrial Functional Genomics \\ Immanuel Kant Baltic Federal University \\ Kaliningrad, Russia \\ Okinawa Institute of Science and Technology \\ Okinawa, Japan \\ ORCID: 0000-0002-6203-9977 \\ Konstantin Gunbin \\ Institute of Cytology and Genetics SB RAS \\ Novosibirsk, Russia \\ Immanuel Kant Baltic Federal University \\ Kaliningrad, Russia \\ genkvg@gmail.com
}

\begin{abstract}
Population structure is an important factor driving genetic changes. Comparing social (termites) and nonsocial (cockroaches) insects we found the difference in their mitochondrial mutational spectrum: transition transversion ratio (ts/tv) is higher in termites compared to cockroaches suggesting potential influence of population structure on mutational patterns.
\end{abstract}

\section{Keywords - mtDNA, Ts/Tv ratio, termites}

Introduction

\section{Ts/Tv ratio in different species}

The transition transversion ratio ( $\mathrm{Ts} / \mathrm{Tv}$ ratio) is very high in human mitochondrial DNA and varies significantly between different species, families, and orders [1], but the reason for this difference remained unknown. Recently it was suggested that the mitochondrial mutational spectrum is associated with cellular and organismal longevity [2]. It was also shown during mutation accumulation experiments that the pattern of mitochondrial mutations is different in four model organisms [3]. So, both polymorphism data and mutation accumulation experiments tell us about differences in the mutational spectrum of mtDNA across species and lineages.

\section{Termites and cockroaches}

Termites are eusocial insects that evolved from cockroaches of order Blattodea. They are among the most abundant terrestrial organisms and play an important role in decomposition and nutrient recycling, as they are able to digest lignocellulose [4].

Colonies of termites can range in their size from several hundred to millions of individuals. Fertile females of the colonies called "queens" can live dozens of years. It's especially pronounced in "higher" termites, which tend to have bigger colonies and more long-lived queens.

All that makes termites the perfect object to study the influence of population structure and longevity on the mitochondrial mutational spectrum.

\section{Inter-species comparisons}

We collected all mitochondrial protein-coding genes for 98 cockroaches and 402 termites species, reconstructed within-species phylogeny using sequences from sister species as outgroups, reconstructed sequences at each

\author{
Konstantin Popadin \\ School of Life Sciences \\ Ecole Polytechnique Federale de Lausanne \\ Lausanne, Switzerland \\ Center for Mitochondrial Functional Genomics \\ Immanuel Kant Baltic Federal University \\ Kaliningrad, Russia \\ konstantinpopadin@gmail.com \\ Thomas Bourguignon \\ Okinawa Institute of Science and Technology \\ Okinawa, Japan \\ Thomas.Bourguignon@oist.jp
}

internal node, derived a list of polarized single-nucleotide substitutions between the nodes, and, focusing on the most neutral synonymous fourfold degenerate sites, counted the observed frequencies of twelve types of nucleotide substitutions.

We calculated the Ts/Tv ratio for every species and found an increased ratio in termites compared to cockroaches (Fig. 1), that might be explained by different generation lengths of the compared groups. Moreover when we split termites into the lower and the higher ones we see a tendence for concordant increase in Ts/Tv from shortlived lower termites to more long-lived higher termites.

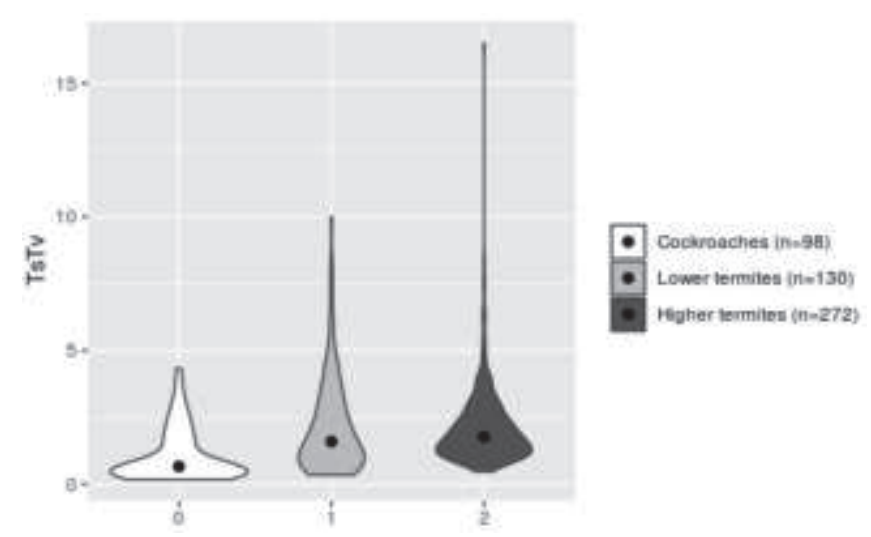

Fig. 1. Ts/Tv ratio for cockroaches, lower and higher termites

\section{Discussion}

We analyzed the mutational spectrum of termites and cockroaches and found an increased $\mathrm{Ts} / \mathrm{Tv}$ ratio in termites. A similar trend was previously found for mammals: longlived mammals have a higher Ts/Tv ratio compared to shortlived [2]. Indeed, termite queens live longer than cockroaches but the presence of secondary reproductives and social structure make mtDNA dynamics in termites more complicated.

Rising the lower boundary of $\mathrm{Ts} / \mathrm{TV}$ ratio from cockroaches to higher termites pushed us to another tempting explanation: this change can be explained by a change in ratio between purines and pyrimidines in nearly 
neutral sites. This process can be driven by change in ROS influence. Obviously, ROS is more important, the higher the life expectancy of the body is.

In addition to previous analyzes, we plan to look deeper into different types of mutations, we also intend to analyze nuclear genes to differentiate mitochondria-specific and common mutation patterns.

\section{Acknowledgment}

We thank OIST Scientific Computing \& Data Analysis Section, as most of the analyzes were made on cluster Sango at OIST.

This work has been supported by the 5 Top 100 Russian Academic Excellence Project at the Immanuel Kant Baltic
Federal University, by the Russian Foundation for Basic Research grant 18-29-13055.

\section{References}

[1] Belle, E. M., Piganeau, G., Gardner, M., \& Eyre-Walker, A. "An investigation of the variation in the transition bias among various animal mitochondrial DNA." Gene 355 (2005): 58-66.

[2] Mikhaylova, Alina G., et al. "Mitochondrial mutational spectrum provides an universal marker of cellular and organismal longevity." bioRxiv (2019): 589168.

[3] Montooth, Kristi L., and David M. Rand. "The spectrum of mitochondrial mutation differs across species." PLoS biology 6.8 (2008).

[4] Jouquet, P., Traoré, S., Choosai, C., Hartmann, C., \& Bignell, D. "Influence of termites on ecosystem functioning. Ecosystem services provided by termites." European Journal of Soil Biology 47.4 (2011): 215-222. 